\title{
An Assessment of Limit Cycle Oscillation Dynamics Prior to L-H Transition
}

\author{
Kimitaka ITOH ${ }^{1,2)}$, Sanae-I. ITOH ${ }^{2,3)}$ and Akihide FUJISAWA ${ }^{2,3)}$ \\ ${ }^{1)}$ National Institute for Fusion Science, Toki 509-5292, Japan \\ ${ }^{2)}$ Itoh Research Center for Plasma Turbulence, Kyushu University, Kasuga 816-8580, Japan \\ ${ }^{3)}$ RIAM, Kyushu University, Kasuga 816-8580, Japan
}

(Received 27 August 2013 / Accepted 29 October 2013)

\begin{abstract}
In this article, experimental observations of limit cycle oscillations (LCO) that precede L-to-H transition are discussed. Issues are: (1) the existence of zonal flows, (2) spatio-temporal evolutions of turbulence intensity, and (3) periodic generations/decays of mean radial electric field and density. The role of Reynolds stress to accelerate the LCO flow is also addressed. The propagation of changes of the density gradient and turbulence amplitude into the core is commented. Varieties in experimental reports on these issues are explained, and possible origins of different interpretations are discussed. Problem definitions for the future research for resolution are presented.
\end{abstract}

(c) 2013 The Japan Society of Plasma Science and Nuclear Fusion Research

Keywords: LH-transition, limit cycle oscillation, zonal flow, electric field bifurcation

DOI: $10.1585 /$ pfr. 8.1102168

\section{Introduction}

After the discovery of transition to the H-mode [1], intensive work has been done to understand the mechanisms that induce the transition to the improved confinement state. Central idea to model the H-mode transition is the fundamental role of radial electric field (Er) in the transition and the suppression of turbulence [2,3]. Although the strong and localized radial electric field in the transport barrier has been confirmed experimentally [4,5] and the suppression of turbulence has also been observed [6-9], the physics mechanisms of $\mathrm{H}$-mode have not yet been quantitatively understood.

The validation of models demands the measurement of nonlinear force on plasmas that causes the transition. The nonlinear force can be measured by observing the dynamical change of radial electric field. Along this line of thought, the study of limit cycle, which can possibly occur near the transition boundary, has been investigated theoretically. Fundamental mechanisms of the edge-localized modes (ELMs) $[10,11]$ in ditherling $\mathrm{H}$-mode have been discussed; that is, the mechanism has explained in terms of the LCO among mean Er and transport [12]. Studies of the zonal flow [13] have been in progress, and another type of the limit cycle among zonal flow and turbulence was proposed [14]. Extension of the study of dynamics from the point model to that of one-dimensional spatial profile has also been developed $[15,16]$. Experimental observations that focus on identifying the nonlinear mechanism were reported in the case of internal transport barrier of CHS [17]. In this case, nonlinear force is dominated by neoclassical process, and the observed nonlinear force was found to be

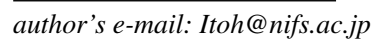

consistent with theoretical predictions.

In the last decade, simultaneous measurements of electric field and turbulence with high space-time resolution have been realized. The methods such as HIBP (heavy ion beam probe) [18-20], Doppler Back Scattering (DBS) measurement [21, 22], GPI (gas puff imaging) [23, 24], ALP (array of Langmuir probes) [25-28] are widely used in experiments. The radial electric field is interpreted in cases of DBS, GPI and ALP with suitable assumptions, while it is directly measured by HIBP. Simultaneous measurements of electric field and turbulence have made a noticeable advancement of physics of plasma turbulence recently [29-31].

In order to study the nonlinear mechanism associated with the $\mathrm{H}$-mode transition, experimental studies have been made on the limit cycle oscillation (LCO) near transition boundary in parameter space, i.e., those for the $\mathrm{H}$-mode in tokamaks [12, 23, 28, 32-41] and for internal transport barrier for helical plasmas [42, 43]. (The observation on TJ-II is often referred to as 'H-mode'. However, we here classify it as an internal transport barrier by two reasons. First, the distance of the barrier from the surface is much longer than the spatial width of the localized strong radial electric field at the barrier. Second, owing to the neoclassical mechanism, the observed phenomena in TJ-II have analogy to internal transport barrier phenomena in CHS.) Prototypical examples of limit cycle oscillations are illustrated in Fig. 1. Figure 1 (a) shows an example of the periodic burst of $\mathrm{H} \alpha$ signal (JFT-2M), which was observed in an early phase of the research. Simultaneous measurement of turbulence intensity and radial electric field on TJ-II is quoted in Fig. 1 (b), which forms a cyclic trajectory on the plane of radial electric field and 

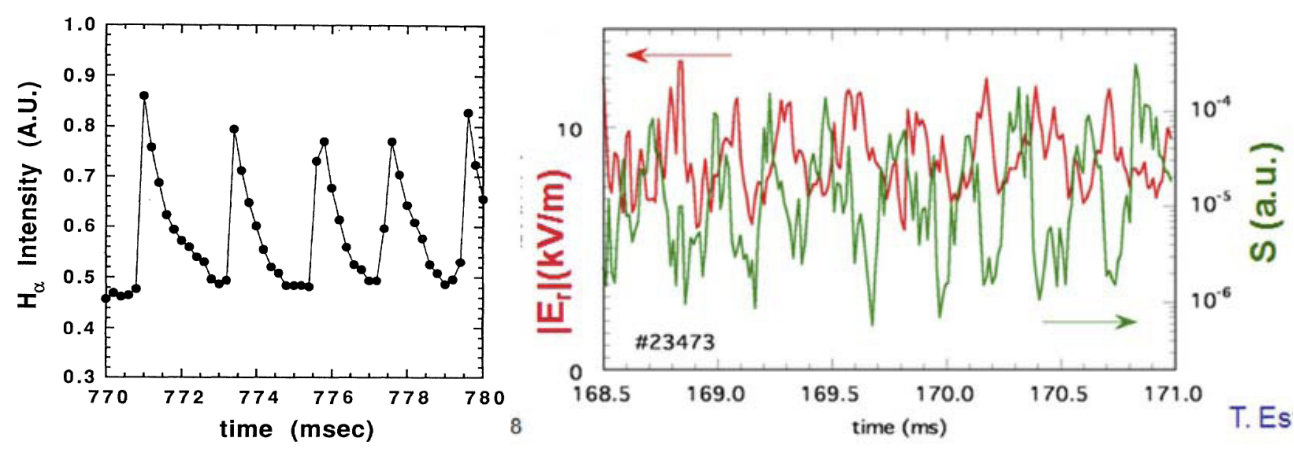

Fig. 1 Self-sustained oscillations in JFT-2M (adopted from [12]) (a) and that from TJ-II (adopted from [43]) (b).
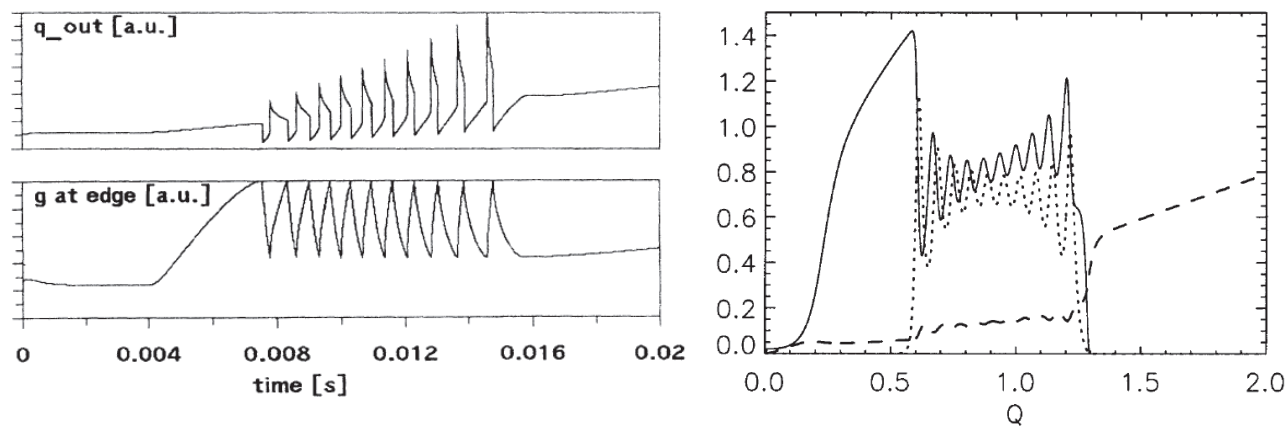

Fig. 2 Results of theoretical models. Model I (left) [12,33], and model II (right) [14]. In the case of model I, heat flux (top) and gradient (bottom) are shown (adopted from [33]). The right figure shows the evolution of amplitude of turbulence (solid line), zonal flow shear (dotted line), and the gradient of ion pressure (dashed line) as a function of input power $\mathrm{Q}$ that increases in time $t$ (adopted from [14]).

turbulence intensity. As the number of observations increases, varieties have also become noticeable. Many of these discussed the hypothesis that the turbulence drives zonal flows, which later suppress the turbulence. However, the assessment of the causality between turbulence and flow (i.e., which changes first) differs in couple of articles. The identification of zonal flows in LCO has not yet been completed, except some limited cases. The origin of the oscillatory $E \times B$ flow must be identified. The oscillation of radial electric field and turbulence intensity might give a clue to understand the other outstanding mystery, i.e., the rapid change of core confinement after the onset of L-H transition $[44,45]$.

It is a time to make a critical assessment on the experiments of limit cycle oscillations (LCO), in order to make a firm basis for the understanding of the physics of L-H transition. In this article, we make an assessment of recent experimental observation on LCO phenomena. Various discrepancies are put in order, and central issues in varieties in interpretations are formulated in Sec. 2. The problem definitions for resolution (and possibly unification) are explained in Sec.3. Possible further developments in the study of LCO dynamics are discussed in Sec. 4.

\section{Survey of Experimental Observa- tion}

\subsection{Background of LCO dynamics}

It might be useful to revisit models of limit cycle oscillations, which are caused by nonlinearities in the transition mechanism. In theoretical models, turbulence and turbulent transport are considered to be a nonlinear function of electric field filed (either mean radial electric field or zonal flows). In the first model, model I, [12], the time-dependent Ginzburg-Landau (TDGL) type equation was modeled for the evolution of mean radial electric field. On the other hand, in the model II, VolterraLotca type equation was analyzed for the model with zonal flow, [14]. In the former model, the trajectory in the space of \{global parameter and turbulence intensity shows a counter-clockwise (CCW) limit cycle. On the other hand the trajectory in model II shows a clockwise (CW) limit cycle. (In this article, we choose a convention to plot the turbulence intensity in the vertical axis). The other difference is that, in the model I, the electric field is employed as a dynamical parameter, while the squared intensity of zonal flow electric field is taken as a variable in the model II. It has been pointed out, theoretically, that two types of Limit cycles (CCW and $\mathrm{CW}$ ) are possible to occur. 
Results of theoretical modelling are quoted in Fig. 2.

The other issue is the rate of acceleration in the limit cycle oscillation. The rate of acceleration has been predicted by theories. The origins that can generate (decrease) radial electric field was summarized as $[46,47]$

$$
\frac{\varepsilon_{0} \varepsilon_{\perp}}{e} \frac{\partial}{\partial t} E_{r}=\Gamma_{e-i}^{\mathrm{anom}}-\Gamma_{i}^{\mathrm{lc}}-\Gamma_{i}^{\mathrm{bv}}-\Gamma_{i}^{\nu \nabla v}-\Gamma_{i}^{\mathrm{cx}}-\Gamma_{i}^{\mathrm{NC}}+\Gamma_{e}^{\mathrm{NC}},
$$

where $\varepsilon_{\perp}$ is the relative dielectric constant (the ratio between the dielectric constant and that of the vacuum $\varepsilon_{0}$ ). The terms in the RHS of Eq. (1) stand for the processes like convective loss of waves (first term), ion orbit loss (second term), neoclassical bulk viscosity (third term), turbulent Reynolds stress (fourth term), loss by charge exchange (fifth term), and other neoclassical processes for ions and electrons, respectively. The relative dielectric constant is given as

$$
\varepsilon_{\perp}=1+2 q^{2}
$$$$
\text { (plateau regime), }
$$

and

$$
\varepsilon_{\perp}=1+1.6 q^{2} / \sqrt{\varepsilon} \quad \text { (banana regime) }
$$

respectively. As is explained in reviews [13,46-48], the dielectric constant is higher in toroidal plasmas, in comparison with cylindrical (or slab) plasmas. This has been explained in two ways. In one way of explanation (the kinetic description of particle motion), the enhanced dielectric constant is owing to the larger deviation of particle orbits from magnetic surfaces (by toroidal drift) [49]. The longer excursion of particles across magnetic surfaces makes the polarization drift larger, so that the dynamic change of radial electric field is more easily screened. In alternative way of explanation (the fluid description), the poloidal $E \times B$ flow is associated with the toroidal return flow. This is because the $E \times B$ velocity in poloidal direction has a finite divergence, which must be compensated by the return flow along the field line for the case of $\omega<\omega_{\mathrm{GAM}}$. The direction of this toroidal component is opposite between inside and outside of torus (being analogous to the Pfirsch-Schlueter current). As a result, the low-frequency $E \times B$ flow takes a form of helical flow (see illustration in Fig. 3). The effective inertia for poloidal acceleration increases.

If one focuses on the response of poloidal motion for the frequency range below GAM frequency, $\omega<\omega_{\mathrm{GAM}}$, against the turbulent Reynolds stress and collisional damping, an equation of motion for the perturbation takes a form

$$
\varepsilon_{\perp} \frac{\partial}{\partial t} V_{\theta}=-\nabla\left\langle\tilde{v}_{r} \tilde{v}_{\theta}\right\rangle-v_{\text {damp }} V_{\theta}
$$

Comparing the magnitude of the inertial term (LHS) to the driving (the first term of RHS), we have a necessary condition that the first term in RHS can sustain the stationary oscillation of poloidal flow velocity as

$$
\frac{\left|V_{\theta} \nabla\left\langle\tilde{v}_{r} \tilde{v}_{\theta}\right\rangle\right|}{\omega_{\mathrm{LCO}}\left|V_{\theta}\right|^{2}}>\varepsilon_{\perp}
$$

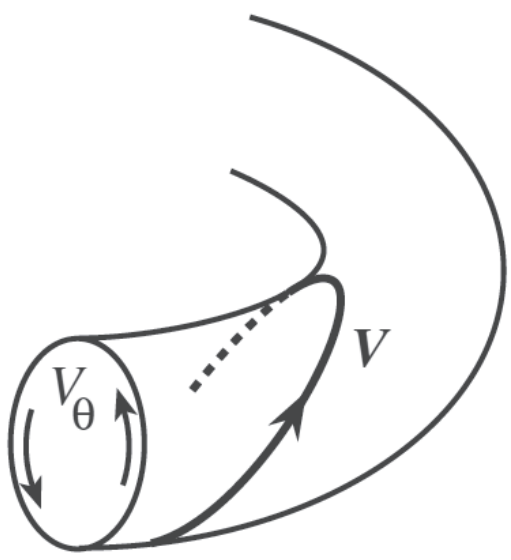

Fig. 3 Flow pattern for low frequency zonal flows (adopted from [13]).

This condition is more stringent than the case of cylindrical (slab) plasmas, because $\varepsilon_{\perp}>1$ holds.

\subsection{Observations of limit cycle oscillations near transition boundary}

Prototypical examples of limit cycle oscillations are illustrated in Fig. 1. This type of periodic bursts of $\mathrm{H}_{\alpha} / \mathrm{D}_{\alpha}$ signals and modulation of turbulence intensity has been observed in many experimental devices. Various reports are summarized in Table 1. This table shows the method of diagnostics, the frequency of periodic oscillations, radial location where the LCO occurs, role of mean radial electric field, presence of zonal flows and the causality among radial electric field and turbulence intensity. In early stage (1990's), measurement of turbulence was limited. This table covers the LCO in the L-H transition dynamics as well as that related with internal transport barrier. Owing to the neoclassical mechanism, which drives the radial electric field, the observed phenomena in TJ-II should have a deep commonality with internal transport barrier phenomena in CHS [50], which has clearly demonstrated the impact of zonal flow on turbulence intensity in conjunction with the formation/decay of transport barriers.

Surveying the Table 1, one sees varieties in experimental reports. In particular, varieties in interpretations are seen about the causality between the electric field and turbulence, and about the presence of zonal flows. We make an assessment on the following essential points:

(1) Order in the cycle - Which changes first? The electric field (mean field or zonal flows) or turbulence intensity?

(2) Presence of zonal flow?

(3) Origin of radial electric field (mean field or zonal flows) - Does turbulence Reynolds stress suffice?

\subsection{Varieties in reports}

\subsubsection{Order in the cycle}

The temporal order among changes of electric field 
Table 1 Experimental reports on limit cycle oscillations near the transition boundary. Direction of cyclic dynamics, CW and CCW, is defined on the plane, where the absolute value of radial electric field is in the horizontal axis, and the turbulence intensity is in the vertical direction.

\begin{tabular}{|c|c|c|c|c|c|c|c|}
\hline Device & $\begin{array}{l}\text { Ref } \\
\text { (year) }\end{array}$ & $\begin{array}{l}\text { loca- } \\
\text { tion }\end{array}$ & $\begin{array}{l}\text { LCO } \\
\text { freq. }\end{array}$ & $\begin{array}{l}\text { Diag- } \\
\text { nos. }\end{array}$ & mean $\mathrm{Er}$ & zonal flows & $\begin{array}{l}\text { phase rela-tion } \\
\text { in LCO }\end{array}$ \\
\hline JFT-2M & [12] (91) & edge & $0.5 \mathrm{kHz}$ & & perhaps & not measured & ccW \\
\hline ASDEX-U & [33] (94) & edge & $\sim 1.5 \mathrm{kHz}$ & & perhaps & not measured & ccW \\
\hline NSTX & {$[23](10)$} & $\begin{array}{l}\text { edge } \\
\text { SoL }\end{array}$ & $\sim 3 \mathrm{kHz}$ & GPI & ? & $?$ & $\begin{array}{l}\text { SoL density - } \\
\text { Vp: anti corre- } \\
\text { lation CCW? }\end{array}$ \\
\hline ASDEX-U & [35] (11) & edge & $\sim 2.5 \mathrm{kHz}$ & DBS & $Y$ & GAM & \\
\hline EAST & [36] (11) & edge & $4 \mathrm{kHz}$ & Probe & & $\begin{array}{l}\text { ZF: energy } \\
\text { balance }\end{array}$ & $\begin{array}{l}\text { anti- } \\
\text { correlation }\end{array}$ \\
\hline DIIID & {$[37](12)$} & edge & $\sim 2 \mathrm{kHz}$ & DBS & $Y$ & $\begin{array}{l}\text { ZF: radial wave } \\
\text { number }\end{array}$ & $\begin{array}{l}\mathrm{CW} \text { and } \\
\text { variations }\end{array}$ \\
\hline DIIID & {$[38](13)$} & edge & $\sim 2.5 \mathrm{kHz}$ & Probe & maybe & $\begin{array}{l}\text { ZF: energy } \\
\text { balance }\end{array}$ & $\mathrm{CW}$ \\
\hline HL-2A & [39] (13) & edge & $2 \sim 3 \mathrm{kHz}$ & Probe & $Y$ & perhaps No & $\mathrm{CCW}$ and $\mathrm{CW}$ \\
\hline JFT-2M & {$[41](13)$} & edge & $4.5 \mathrm{kHz}$ & HIBP & $Y$ & No & CCW \\
\hline TJ-II & [43] (10) & $\mathrm{r} / \mathrm{a}<0.8$ & $\sim 2 \mathrm{kHz}$ & DBS & & maybe & $\mathrm{CW}$ \\
\hline CHS & {$[42](98)$} & $\mathrm{r} / \mathrm{a} \sim 0.4$ & $\sim 0.5 \mathrm{kHz}$ & HIBP & $\begin{array}{l}\text { leading } \\
\text { bifurcation }\end{array}$ & not measured & \\
\hline CHS & {$[50](06)$} & $\mathrm{r} / \mathrm{a} \sim 0.4$ & $\begin{array}{l}\text { low } \\
\text { freq. }\end{array}$ & HIBP & \begin{tabular}{|l|} 
leading \\
bifurcation
\end{tabular} & zonal flow & $\begin{array}{l}\text { ZF-turb.: } \\
\text { anti-corr. }\end{array}$ \\
\hline
\end{tabular}

(a)

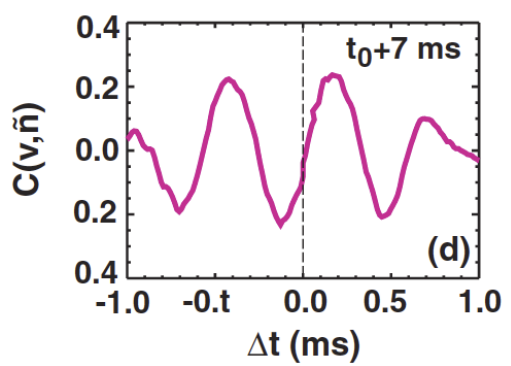

(b)

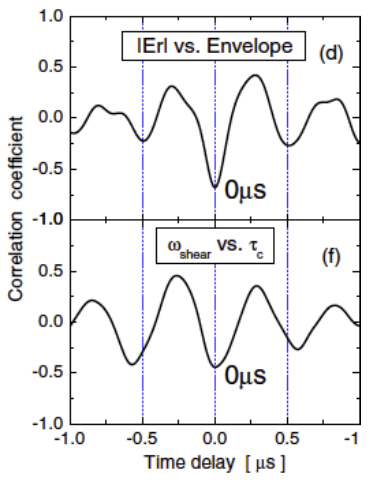

(c)

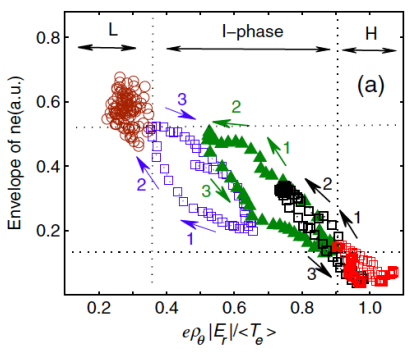

Fig. 4 Phase relations between electric field and fluctuations: DIII-D (adopted from [37]) (a) and EAST (adopted from [36]) (b). The change of phase relation which was observed on HL-2A (adopted from [39]) (c).

and turbulence intensity in TJ-II [43] has stimulated the idea that the limit cycle might be caused by the nonlinear dynamics in the system of zonal flow - turbulence. That is, the temporal order is consistent with the theoretical picture that the high intensity of turbulence causes the growth of radial electric field of zonal flows, which later suppresses the turbulence. (Once the turbulence is suppressed, the radial electric field starts to decrease due to the collisional damping.) Owing to the fact that the order can give a clue to identify the causality, the temporal order in the limit cycle has been studied intensively. The outcome is that the direction in limit cycle dynamics looks to differ from device to device (Fig. 4). In the report [37], observation on DIII-D tokamak shows that the electric field changes with the phase delay with respect to the turbulence intensity, confirming the observation on the TJ-II (Fig. 4 (a)). However, EAST tokamak reported that there is no phase delay between them, that is, the fluctuation intensity changes oppositely with respect to the radial electric field [36] (Fig. 4(b)). Various results on the direction of cycle have been reported, even from one device [37, 51]. In addition, an opposite direction of circulation was reported from recent analyses on data from HL-2A [39, 40] (Fig. 4 (c)) and JFT-2M [41]. 

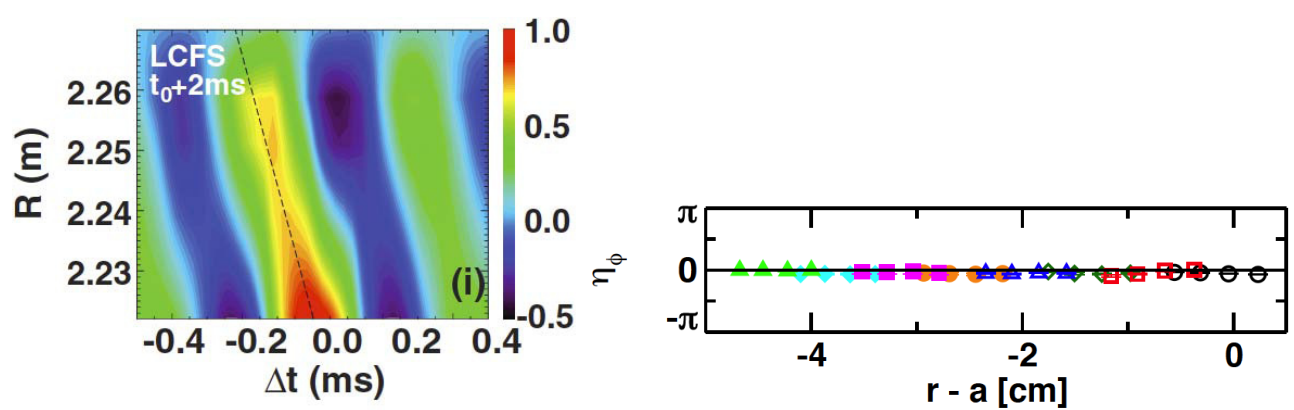

Fig. 5 Wave number of electric perturbation in LCO. The result in DIII-D (adopted from [37]) shows a finite radial wave number $~$ $0.7 / \mathrm{cm}$ (a). The phase delay of potential perturbation with respect to the $\mathrm{H}_{\alpha}$ oscillation is shown as a function of radius in JFT-2M plasmas (adopted from [41]) (b). In this case, very small radial wave number is obtained (b).

\subsubsection{Presence of zonal flow}

The conclusion of the presence of zonal flow waits further analyses.

First, the report from ASDEX-U has identified the existence of GAMs [35]. This was concluded by the frequency of oscillations. The study of JFT-2M has shown that GAMs appear in the L-mode but disappears before the LCO happens [41]. In other experiments, GAMs were not reported in LCO. Therefore it is evident that there are (at least) two types of limit cycle state (i.e., with GAMs and without GAMs). The phase diagram for the appearance of GAMs has been discussed in literature [52], so that assessment in such a phase diagram will be a productive approach.

Next, although zonal flows are conjectured in many reports, the evidence for the presence of zonal flows in LCO plasmas was limited so far. In this article, we employ the criteria for the zonal flows as:

(i) ZFs are constant on magnetic surface, and are not associated with electron density perturbation (in the lowest order of ion gyroradius effect),

(ii) ZFs have substantial radial wavenumber,

(iii) ZFs are driven primarily by turbulence Reynolds stress.

These criteria are chosen following the review [13].

To the best of authors' knowledge, there has not been a report, which confirmed that the observed oscillatory electric field satisfies these three criteria simultaneously. In supporting the presence of zonal flow, DIII-D has shown that criteria (i) and (ii) are satisfied [37]. The radial wave number, $\sim 0.7 / \mathrm{cm}$, was reported (Fig. 5 (a)). On the other hand, further analysis seems necessary for judging the criteria (iii). It is claimed that the magnitude of the drive by turbulence Reynolds stress is consistent with what is necessary to sustain the observed $E \times B$ flow [38]. However, the care is necessary for the dielectric constant in toroidal plasmas [46-49], and further studies are required. The same applies to the study on EAST. The report [36] claims that zonal flows are identified from the consideration of energy balance of $E \times B$ flow. Nevertheless, criterion (ii) is not confirmed in the report. The dielectric constant is not investigated completely, either. In contrast to these reports, the analysis on JFT-2M has shown that both the criteria (ii) and (iii) are not satisfied, i.e., there are no zonal flows in the LCO [41]. It is possible that (a) there are two types LCO (being discriminated by the presence of zonal flows), or that (b) more precise study in future will resolve present apparent differences. The more detailed study on the cause of $E \times B$ flows is necessary.

\subsubsection{Origin of radial electric field (mean field or zonal flows)}

As is mentioned in preceding subsection, turbulence Reynolds stress is often considered to drive the observed oscillating $E \times B$ flow velocity. If one quotes the dynamical equations from [38], they have the structure as

$$
\begin{aligned}
& \frac{\partial}{\partial t} \tilde{v}_{\perp}^{2}=\gamma_{\mathrm{eff}} \tilde{v}_{\perp}^{2}-\gamma_{\mathrm{cesor}}^{\mathrm{pl}} \tilde{v}_{\perp}^{2}-\left\langle\tilde{v}_{r} \tilde{v}_{\theta}\right\rangle^{\prime} V_{E \times B}^{\mathrm{LF}}, \\
& \frac{\partial}{\partial t} V_{E \times B}^{\mathrm{LF}}{ }^{2}=\left\langle\tilde{v}_{r} \tilde{v}_{\theta}\right\rangle^{\prime} V_{E \times B}^{\mathrm{LF}}-v_{\mathrm{LF}} V_{E \times B}^{\mathrm{LF}}{ }^{2},
\end{aligned}
$$

where $\tilde{v}_{\perp}^{2}$ is the turbulence energy density (per unit mass) and $V_{E \times B}^{\mathrm{LF}}{ }^{2}$ is the flow energy density (per unit mass). In this system of equations, the relative dielectric constant is taken to be unity. The measured value of the Reynolds stress is substituted into the work done by the stress, and Ref. [38] commented that the work $\left\langle\tilde{v}_{r} \tilde{v}_{\theta}\right\rangle^{\prime} V_{E \times B}^{\mathrm{LF}}$ plays a substantial role in inducing the increase of flow energy. A similar argument was applied to the EAST tokamak [36].

In the analysis of JFT-2M data, the enhancement of dielectric constant in toroidal plasmas $\left(\varepsilon_{\perp}=1+2 q^{2}\right.$ in plateau regime) is taken into consideration. From the equation of motion, the oscillatory velocity, which is induced by the oscillatory Reynolds stress at the angular frequency of $\omega_{\mathrm{LCO}}$, is evaluated as

$$
\delta\left|V_{E \times B}\right| \sim\left|\Pi_{r \theta}\right| L^{-1} \varepsilon_{\perp}^{-1} \omega_{\mathrm{LCO}}^{-1} .
$$

Here, the magnitude of force in poloidal direction per unit mass, $\partial \Pi_{r \theta} / \partial r$, is evaluated as $\left|\Pi_{r \theta}\right| L^{-1}$, and $L \sim 1 \mathrm{~cm}$ is 


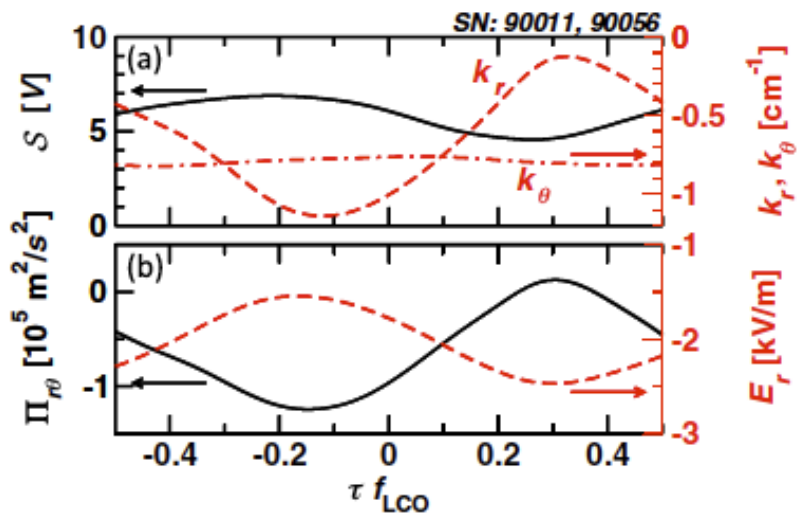

Fig. 6 periodic changes of Reynolds stress and electric field at LCO frequency (JFT-2M) (adopted from [41]).

used for the scale length of radial gradient of $\Pi_{r \theta}$. Figure 6 illustrates the periodic changes of various quantities at the frequency of limit cycle oscillation. Modulation amplitude of $\Pi_{r \theta}$ is evaluated as $\left|\Pi_{r \theta}\right| \sim 5 \times 10^{4} \mathrm{~m}^{2} / \mathrm{s}^{2}$. By use of parameters $\left|\Pi_{r \theta}\right| L^{-1} \sim 5 \times 10^{6} \mathrm{~m} / \mathrm{s}^{2}, \omega_{\mathrm{LCO}} \sim 3 \times 10^{4} \mathrm{~s}$, and $\varepsilon_{\perp} \sim 20$ for $q \sim 3$, one has the amplitude of modulation $\delta\left|V_{E \times B}\right| \sim 10 \mathrm{~m} / \mathrm{s}$. This contribution is much smaller than the observed modulation of $E \times B$ velocity in the LCO $(\sim 500 \mathrm{~m} / \mathrm{s})$. Another important finding is the phase between the Reynolds stress force and the acceleration of the velocity. Figure 6 demonstrates that the phases in LCO of Reynolds stress force and the $E \times B$ velocity are close to each other, i.e., the phase difference between force and acceleration is close to $\pi / 2$. This also indicates that the Reynolds stress force is not the main origin of acceleration in LCO. The conclusion on JFT-2M observation was that the drive by turbulence Reynolds stress remains partial contribution for the oscillatory $E \times B$ oscillation.

\section{Resolutions and Possible Unifica- tion}

We here discuss a few physics issues, which might induce variances of the statements on the LCO observation on various devices. Studies of experimental data by noting these aspects will be a relevant problem definition for the future experimental research.

\section{1 $E \times B$ flow and fluctuations}

In order to analyze the temporal order of changes (in electric field and turbulence intensity), one must carefully consider whether the phase of zonal flow (or temporally varying mean electric field) is resolved or the long time average of zonal flow intensity is followed. It looks that there is confusion in comparing the radial electric field (either mean electric field or zonal flow electric field) with the model picture of dynamical evolution. Figure 7 illustrates the short-time dynamics as well as the long-time dynamics of the zonal flow and drift wave fluctuations. In a short

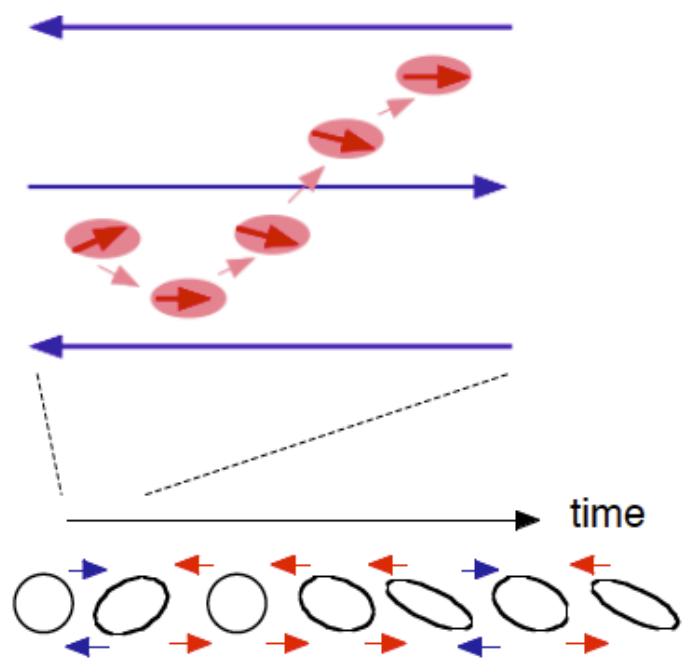

Fig. 7 the short-time dynamics (top) as well as the long-time dynamics (bottom) of the zonal flow and drift wave fluctuations (adopted from [13]). The ellipse stands for packets of drift waves, and arrows indicate zonal flows. Bold arrow on wave packet denotes the wave vector.

time (which is shorter than the auto-correlation time of zonal flows), the sapatio-temporal phase of electric field, which fluctuations feel, is well defined (Fig. 7 top). In contrast, in a long run, the direction of zonal flow varies in a statistical manner (Fig. 7 bottom).

Review article [13] explains the two limiting cases. In the short time scale (the time scale that resolves zonal flow oscillation), fluctuation is subject to the bounce motion in the trough of the zonal flow [53] as is illustrated in Fig. 7 top. Drift wave fluctuations, described as a quasi-particle in the field of zonal flow, obey the equation of packets motion. Within a decorrelation time of fluctuations, they show the bounce motion in the trough, that is packets are trapped in the trough. As a result of this trapping mechanism, turbulence intensity is predicted to be high at the zonal flow trough.

In the time scale, which is longer than the decorrelation time of drift wave packets, the envelope of the wave intensity is employed. The dynamics of the drift wave intensity is described by Eq. (5a), and this equation shows the sensitivity of the turbulence intensity to the phase of zonal flow. When the turbulence Reynolds stress is induced by the disparate-scale interaction with zonal flow, the stress is proportional to the gradient of the radial electric field, i.e., $\left\langle\tilde{v}_{r} \tilde{v}_{\theta}\right\rangle \propto E_{r}^{\prime}$. Thus, the power absorbed from turbulence by the flow has a relation

$$
\left\langle\tilde{v}_{r} \tilde{v}_{\theta}\right\rangle^{\prime} V_{E \times B}^{\mathrm{LF}} \propto-E_{r} \frac{\partial^{2}}{\partial r^{2}} E_{r} .
$$

This relation indicates that the impact on the turbulence intensity is prominent at the spatial location where $E_{r}$ takes the minimum or maximum.

In the longer space scales, the dynamical behaviour is 


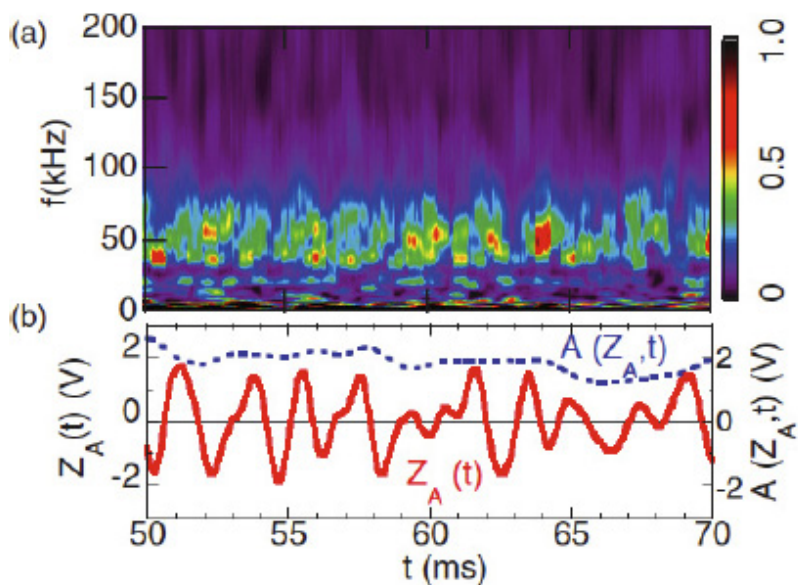

(c)
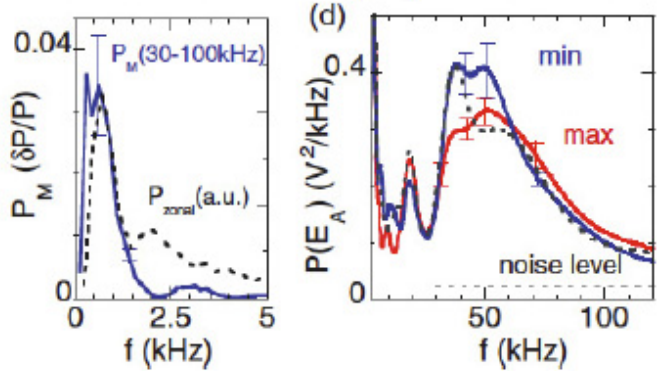

Fig. 8 Turbulence modulation due to low-frequency zonal flows in CHS. (a) Temporal evolutions of wavelet spectrum of electric field fluctuations $E$ defined as $\Delta \phi=E \Delta r$, where $\Delta r$ is the distance between the two spatial channels. Color bar units: $V^{2} \mathrm{kHz}^{-1}$. (b) Evolution of the zonal flows, $Z_{\mathrm{A}}(t)$. Blue dashed line indicates the evolution of zonal flow amplitude using wavelet analysis, Env $\left[Z_{\mathrm{A}} ; t\right]$. (c) FFT spectrum of wavelet power modulation in the frequency range from 30 to $100 \mathrm{kHz}, P_{\mathrm{M}}$. Black dashed line, $P_{\text {zonal }}$, represents the electric field fluctuation in the zonal flow range. (d) Conditional averages of wavelet power spectra around the local maxima and minima of the zonal flow (adopted from [54]).

described by following the evolution of envelope of zonal flows. The envelope is obtained by spatially-averaging the quadratic intensity of zonal flows. After partial integral

$$
-\int \mathrm{d} r E_{r} \frac{\partial^{2}}{\partial r^{2}} E_{r}=\int \mathrm{d} r\left|E_{r}^{\prime}\right|^{2}=\left\langle U^{2}\right\rangle,
$$

where $U$ is the vorticity of zonal flow, the nonlinear interaction between drift wave turbulence and zonal flows has been described, by using the intensities of drift waves zonal flow, as

$$
\begin{aligned}
& \frac{\partial}{\partial t}\langle N\rangle=\gamma_{\mathrm{L}}\langle N\rangle-\gamma_{2}\langle N\rangle^{2}-\alpha\left\langle U^{2}\right\rangle\langle N\rangle, \\
& \frac{\partial}{\partial t}\left\langle U^{2}\right\rangle=-\gamma_{\text {damp }}\left\langle U^{2}\right\rangle+\alpha\left\langle U^{2}\right\rangle\langle N\rangle .
\end{aligned}
$$

Here, $\langle N\rangle$ is the action density of the drift wave turbulence. It should be noted that the quantity $\left\langle U^{2}\right\rangle$ and $\langle N\rangle$ are variables, which change slower than the wavelength of zonal flow oscillations.
These two kinds of responses of turbulence against zonal flow have been confirmed by experiments [54]. Figure 8 (a) illustrates the evolution of zonal flow electric field and spectrum of high frequency turbulence. The high frequency turbulence appears intermittently, and high intensity appears when the zonal flow electric field is negative. This dependence on the sign of zonal flow electric field is quantified by showing the conditional-averaged frequency spectrum. Figure 8 (b) shows the power spectrum of turbulence in the time windows of minima and maxima of zonal flow electric field. The turbulence intensity is unambiguously higher in the time window where zonal flow electric field is negative. In a long-time average, the dependence of turbulence intensity on the zonal flow intensity is different. In Fig. 8 (b), the dotted line indicates the 'envelope' of zonal flow intensity, i.e., the longer-time average of zonal flow intensity, where the integration time for average is longer than the period of zonal flow oscillation. The correlation between the longer-time average of zonal flow intensity and turbulence intensity is demonstrated in [54] too. A monotonic dependence of the turbulence intensity on the squared intensity of zonal flows is shown. In this case, the dependence on the sign of zonal flow electric field is averaged out.

Comparing the prototypical dynamics in two limiting cases, one sees that the interaction in the short time scale is dependent on the sign of the radial electric field, while interactions of the long-time averages are independent of the sign of the zonal flow electric field (because the quantities are averaged in time longer than the period).

\subsection{Acceleration of poloidal flow and nonlin- ear force that induces the H-mode}

The next is an absolute value of nonlinear force that induces the H-mode.

The observation of the temporal dynamics of the radial electric field (LHS of Eq. (1)) can be compared to the various driving terms. The analysis has been performed on the LCO of internal transport barrier of CHS [17] (Fig. 9). From the time derivative of the radial electric field, the net radial current was evaluated. The nonlinear $J_{\mathrm{r}}\left[E_{\mathrm{r}}\right]$ curve was obtained (Fig. 9(b)). This nonlinear $J_{\mathrm{r}}\left[E_{\mathrm{r}}\right]$ curve was compared to the neoclassical component, which is considered to be dominant in helical system CHS. This study has also given an experimental verification that the toroidal dielectric constant is enhanced following Eq. (2a). This approach, which was successful in the case of LCO in CHS internal transport barrier, can also be applicable to the study of LCO near L-H boundary.

\section{Future Works}

Further important researches can be developed by analyzing the LCO dynamics. 
(a)

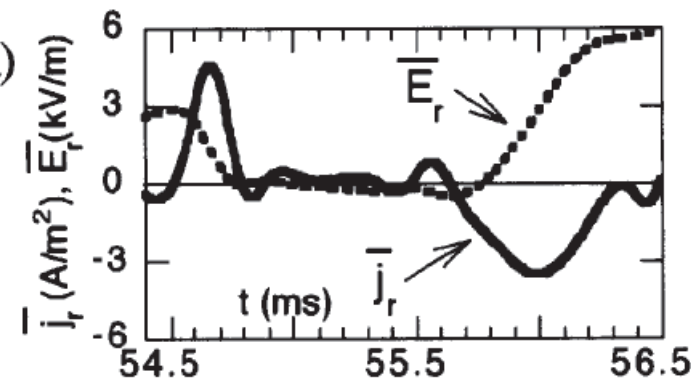

(b)

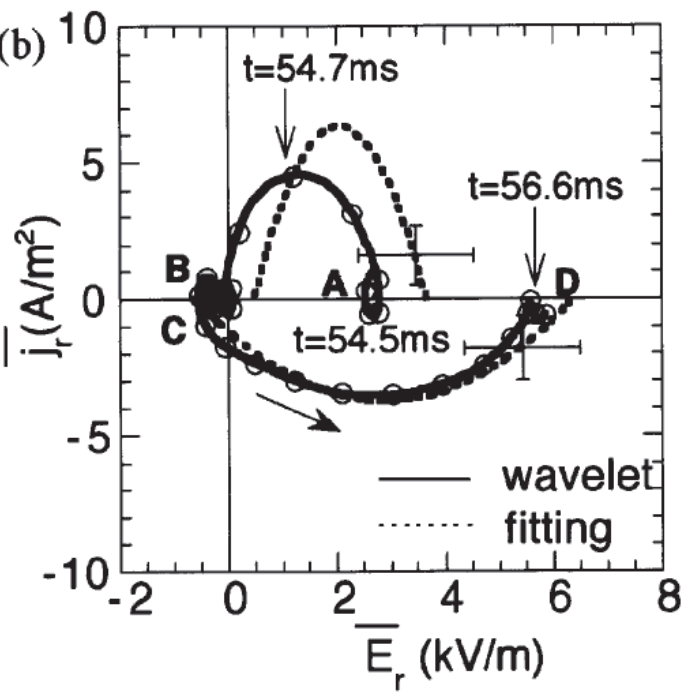

(c)

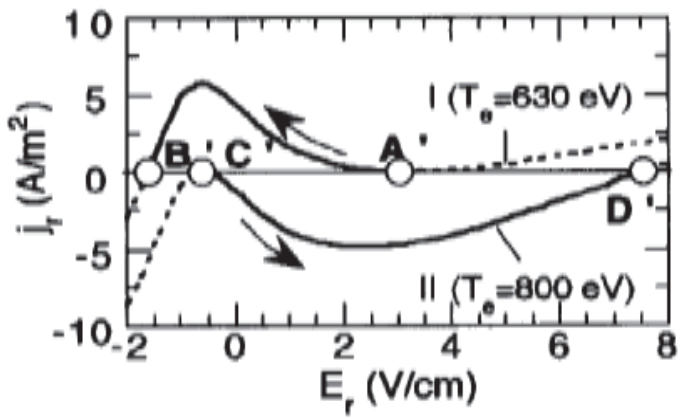

Fig. 9 From the time derivative of the radial electric field, the net radial current was evaluated. (CHS) (adopted from [17]). (a) The solid and dashed lines show the radial current and electric field, respectively. (b) Experimental radial current as a function of radial electric field. The open circles are plotted in every 64 micro seconds. (c) Diagram of calculated radial electric field and radial current using a neoclassical formula. This diagram shows critical conditions to cause transition based on bifurcation.

\subsection{Propagation of the LCO dynamics into the core}

One is the propagation of the response of LCO into the core plasma. The fast change of core transport at the onset of H-mode transition has been known for more than 20 years [44, 45]. Recently, fluctuations that could induce rapid transmission of change of transport have been searched for [55-62]. The response of LCO provides a good opportunity to observe the response of core plasma against the change at the edge. Initial study on the LCO in

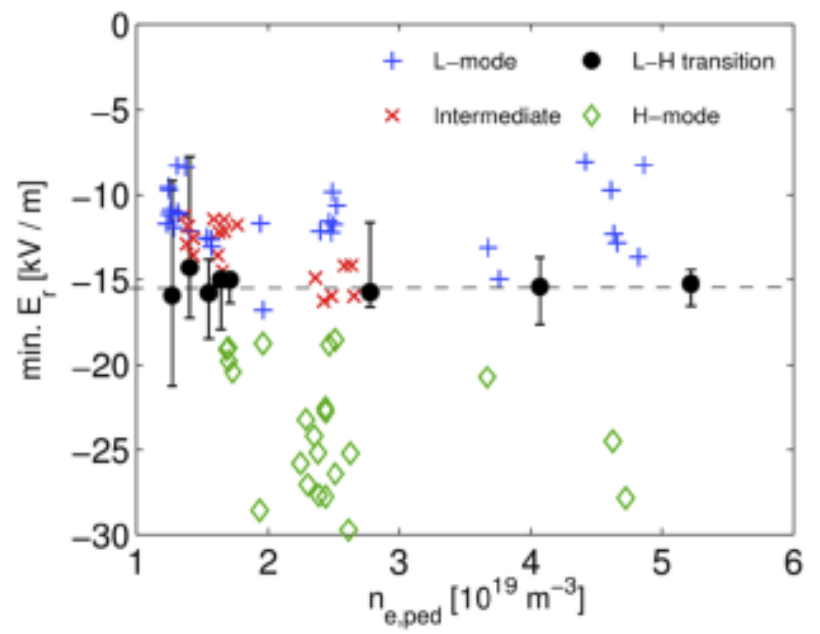

Fig. 10 L-H transition boundary in the space of radial electric filed near edge (adopted from [63]).

JFT-2M has indicated the propagation of the modification of density gradient and turbulence intensity from edge to core [41].

\subsection{Critical condition for the onset of transi- tion}

The other is a study of the critical condition for transition. The study on HL-2A has shown the presence of two types of limit cycle oscillations [39]. One is CW and the other is CCW (See Fig. 4(c)). It was suggested that the bifurcation from one type of limit cycle to the other type occurs if the absolute value of the radial electric field reaches a critical value. This critical value is close to a criterion of normalized radial electric field $e \rho_{\mathrm{p}} E_{r} / T_{\mathrm{i}} \sim \mathrm{O}(1)$ (where the ion temperature is assumed to be equal to the electron temperature). The observations on ASDEX-U indicated that the states of discharge (L-mode, LCO and $\mathrm{H}$-mode) are separated by the minimum radial electric field near edge [63]. In a wide range of plasma density, the critical value of radial electric field was reported to be $E_{r} \sim-15 \mathrm{kV} / \mathrm{m}$ (Fig. 10). For the ion temperature of $T_{\mathrm{i}} \sim 200 \mathrm{eV}$, the normalized radial electric field satisfies the relation $e \rho_{\mathrm{p}} E_{r} / T_{\mathrm{i}} \sim-1$. This magnitude is close to the condition where the bifurcation of radial electric field can occur [2,64-66]. Along this line of thought, an emphasis is made on the importance of ion pressure gradient in [63]. This is because the ion pressure gradient is one of strong driving force for the mean electric field. It should be noted that the radial electric field does not slave to the ion pressure gradient $[2,46,47,64]$. Measurement on JT-60U has also shown that the electric field can change without substantial change of ion pressure gradient [67], as is demonstrated in Fig. 11. Such a sudden change of radial electric field was reported to occur near the condition $e \rho_{\mathrm{p}} E_{r} / T_{\mathrm{i}} \sim 1$. In LCO, the phase difference between the density gradient and radial electric field is also 


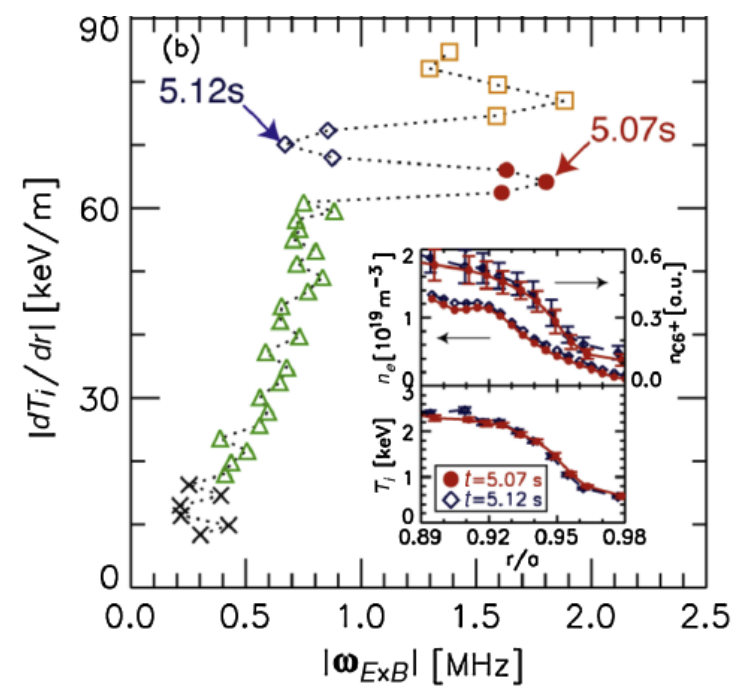

Fig. 11 The relation between the ion temperature gradients (at $\sim 3.9 \mathrm{~cm}$ inside the separatrix) and the $E \times B$ shearing rate (adopted from [67]).

reported [41]. The deviation between the temporal evolution between radial electric field and ion pressure gradient can be important in the transition to the H-mode. The transition without zonal flow is also discussed in [68]. The model in [2] was extended to include the drive by turbulence (the 4th term in the RHS of Eq. (1)) [69]. It is shown that a sudden change of radial electric field was reported to occur near the condition $e \rho_{\mathrm{p}} E_{r} / T_{\mathrm{i}} \sim-1$ for the wide range of collisionarity.

The simulation study is also in progress. In the transport modeling simulations, integrated modeling systems have been applied to the H-mode physics. An integrated simulation model of core and scrape-off-layer (SOL)/divertor transport has been developed, in which a $1.5 \mathrm{D}$ core code TOPICS-IB and a 2D divertor code SONIC are coupled [70]. The dynamic simulation for the $\mathrm{L} / \mathrm{H}$ transition in JT-60SA is carried out by this integrated code using a current-diffusive ballooning mode (CDBM) transport model $[15,47]$ that includes the $E \times B$ shearing effect. The impact of SOL/divertor transport on the $\mathrm{L} / \mathrm{H}$ transition, and the evolution of the core transport after the transition has also been studied. This integrated simulation has indicated that the understanding of the rapid response in the core transport after the transition at the edge [45] is a real challenge for simulations. The other is the transport simulation on L-H transition using CDBM transport model, taking account of toroidal rotation effect (more consistent force balance relation) into the 1.5D integrated transport code TOPICS [71]. The rotation and barrier formation in multi-ion-species plasmas has also been studied. A two-fluid transport code TASK/TX [72] was developed, in which the equations of motion for electrons and ions are directly solved and the rotation and the radial electric field are self-consistently described. The radial profiles of poloidal and toroidal rotations for impurity and bulk ions and that of radial electric field are systematically studied with and without transport barriers [73]. The direct nonlinear simulations have demonstrated some essential features of transition. In the fluid turbulence simulations, in which the neoclassical model for damping of the mean radial electric field is included, the appearance of a strong and localized mean radial electric field at the plasma edge is reproduced $[74,75]$. Emerging result has reported from kinetic simulations. The transition to the internal transport barrier was reported to occur in gyrokinetic simulations, if one introduces a model external source [76].

In analyzing the plasmas in the regime of $e \rho_{\mathrm{p}} E_{r} / T_{\mathrm{i}} \sim$ -1 , more emphasis must be placed on the poloidal structure of the barrier. In this regime, the possibility of poloidal shock has been pointed out [77], for which explanation is developed in Chap.21 of [47]. Two-dimensional structure of the transport barrier has been studied, and the enhanced ion pinch has been discussed $[78,79]$. Transport modeling, which takes into account the toroidal effects, has been developed [80]. The consideration of toroidal dielectric constant has cast a problem in the one-dimensional model, which is based on a dynamics of slab (cylindrical) plasmas. More detailed analysis on the toroidal effects is essential in understanding of the physics of $\mathrm{L}-\mathrm{H}$ transition.

The other approach to better understanding is the detailed study of transition, which is induced by external drives. Studies of the transition physics have been performed in experiments using biased electrodes in the plasma periphery [81, 82]: As the applied voltage was increased, the radial electric field bifurcation occurred at some critical point, manifested by a reduction of the electrode current, an increase of the core density, etc. The spatial profile of the radial electric field at the plasma edge showed a change from a flat one before bifurcation to a peaked one after bifurcation. The peak of the radial electric field was localized at the mid-radius between the electrode and the limiter [82]. Theory of induced bifurcation was developed in Ref. [83], where the radial current was evaluated by the neoclassical radial flux. By employing a model form of the electric conductivity and an anomalous ion viscosity, the radial structure of the electric field was analyzed, and a solitary radial electric field and a transition have been obtained [84]. The bifurcation from the state of a single peak to that with double peaks has been predicted theoretically [85]. Recent experiment on LHD has demonstrated the bifurcation to the state of double peaks of localized radial electric field [86]. The measurement of turbulence is made simultaneously. It was found that the turbulence is localized in the trough of the (positive) radial electric field. An asymmetry of turbulence intensity between the trough and peak of radial electric field is observed. That is, the curvature of the radial electric field plays an important role in the evolution of turbulence. The dependence of suppression of turbulence on curvature can leads the exchange of energy of turbulence between differ- 
ence places via mean/meso radial electric field, which has been observed on a basic experiment device [87].

At this moment, the experimental observations of critical condition have not yet been summarized in terms of the edge radial electric field. The deviation of radial electric field from the component, which is driven by neoclassical process, is one of keys for bifurcation. The dependence of the fluctuation intensity on the sign of radial electric field suggests a possibility that the absolute value of the radial electric field plays important role in determining the transition boundary. Careful study of nonlinear response in LCO will give quantitative understanding for the critical condition of the L-H transition.

\section{Summary}

In this article, we make an assessment of recent experimental observation on LCO phenomena. An assessment is made focusing on the following essential points:

(i) Which changes first? The electric field (mean field or zonal flows) or turbulence intensity?

(ii) Are zonal flows present?

(iii) Does the turbulence Reynolds stress play a central role in driving radial electric field (mean field or zonal flows)?

Surveying the Table 1, one sees varieties in experimental reports. In particular, differences are seen about the causality between the electric field and turbulence, and about the presence of zonal flows. Various different interpretations are put in order, and central issues are formulated. A key to these differences in interpretations is discussed. Several critical conditions for the Reynolds stress to induce oscillatory flow are deduced. The problem definitions are developed. Possible further developments in the study of LCO dynamics are discussed. More detailed analysis on the toroidal effects (e.g., toroidal dielectric constant, possible poloidal shock, etc.) is essential in understanding the physics of L-H transition. Along this line of problem definition for experimental studies, the understanding of $\mathrm{H}$-mode physics will be much deeper in the future.

\section{Acknowledgments}

Authors wish to thank discussion and collaboration with Y. Miura, T. Kobayashi, T. Ido, K. Kamiya, Y. Nagashima, S. Inagaki, K. Ida, A. Fukuyama, P. H. Diamond, K. Hallatschek, G. R. Tynan, U. Stroth, J. Q. Dong, K. J. Zhao, and C. Hidalgo, and supports by M. Mori and Y. Kamada. The discussions at the first, second and 3rd APTWG Meetings (in particular 'call for critical experimental test' by P. H. Diamond at the 2nd APTWG meeting), and at 14th International Workshop on H-mode Physics and Transport Barriers are also acknowledged. This work is partly supported by the Grant-in-Aid for Scientific Research of JSPF, Japan (21224014, 23244113), the collaboration programs of RIAM of Kyushu University, of
JAEA and of NIFS, and by Asada Science Foundation.

[1] F. Wagner et al., Phys. Rev. Lett. 49, 1408 (1982).

[2] S.-I. Itoh and K. Itoh, Phys. Rev. Lett. 60, 2276 (1988).

[3] H. Biglari, P.H. Diamond and P.W. Terry, Phys. Fluids B 2, 1 (1990).

[4] R.J. Groebner et al., Phys. Rev. Lett. 64, 3015 (1990).

[5] K. Ida et al., Phys. Rev. Lett. 65, 1364 (1990).

[6] A. Fujisawa et al., Phys. Rev. Lett. 82, 2669 (1999).

[7] F. Wagner, Plasma Phys. Control. Fusion 39, A23 (1997).

[8] D.A. Koslov and T-10 Team, Nucl. Fusion 41, 1473 (2001).

[9] K.H. Burrel, Rev. Sci. Instrum. 72, 906 (2001).

[10] ASDEX Team, Nucl. Fusion 29, 1959 (1989).

[11] H. Zohm, Plasma Phys. Control. Fusion 38, 105 (1996).

[12] S.-I. Itoh et al., Phys. Rev. Lett. 67, 2485 (1991).

[13] P.H. Diamond, K. Itoh, S.-I. Itoh and T.S. Hahm, Plasma Phys. Control. Fusion 47, R35 (2005).

[14] E.-J. Kim and P.H. Diamond, Phys. Rev. Lett. 90, 185006 (2003).

[15] A. Fukuyama et al., Plasma Phys. Control. Fusion 38, 1319 (1996).

[16] K. Miki and P.H. Diamond, Phys. Plasmas 19, 092306 (2012).

[17] A. Fujisawa et al., Phys. Rev. Lett. 79, 1054 (1997).

[18] T.P. Crowley, IEEE Trans. Plasma Sci. 22, 291 (1994).

[19] A. Fujisawa et al., Rev. Sci. Instrum. 63, 3694 (1992).

[20] T. Ido et al., Nucl. Fusion 46, 512 (2006).

[21] M. Hirsch et al., Plasma Phys. Control. Fusion 43, 1641 (2001).

[22] G. Conway et al., Plasma Phys. Control. Fusion 47, 1165 (2005).

[23] S.J. Zweben et al., Phys. Plasmas 17, 102502 (2010).

[24] I. Shesterikov, Y. Xu, C. Hidalgo, M. Berte, P. Dumortier, M. Van Schoor, M. Vergote, G. Van Oost and the TEXTOR Team, Nucl. Fusion 52, 042004 (2012).

[25] Y. Nagashima et al., Phys. Rev. Lett. 95, 095002 (2005).

[26] K. Zhao et al., Phys. Rev. Lett. 96, 255004 (2006).

[27] Y. Nagashima et al., Rev. Sci. Instrum. 82, 033503 (2011).

[28] M. Xu et al., Phys. Rev. Lett. 108, 245001 (2012).

[29] S.-I. Itoh, Plasma Fusion Res. 4, 038 (2009).

[30] S.-I. Itoh, J. Plasma Fusion Res. 86, 334 (2010) (in Japanese).

[31] A. Fujisawa, Nucl. Fusion 49, 013001 (2009).

[32] S.-I. Itoh, K. Itoh and A. Fukuyama, Nucl. Fusion 33, 1445 (1993).

[33] H. Zohm, Phys. Rev. Lett. 72, 222 (1994).

[34] R.J. Colchin et al., Phys. Rev. Lett. 88, 255002 (2002).

[35] G.D. Conway et al., Phys. Rev. Lett. 106, 065001 (2011).

[36] G.S. Xu et al., Phys. Rev. Lett. 107, 125001 (2011).

[37] L. Schmitz et al., Phys. Rev. Lett. 108, 155002 (2012).

[38] G.R. Tynan et al., Nucl. Fusion 53, 073053 (2013).

[39] J. Cheng et al., Phys. Rev. Lett. 110, 265002 (2013).

[40] K.J. Zhao et al., IAEA Fusion Energy Conf. (San Diego, 2012) EX/7-2Ra.

[41] T. Kobayashi et al., Phys. Rev. Lett. 111, 035002 (2013).

[42] A. Fujisawa et al., Phys. Rev. Lett. 81, 2256 (1998).

[43] T. Estrada et al., EPL 92, 35001 (2010).

[44] K.H. Burrell et al., International Conf. Plasma Phys. Control. Fusion 1, 123 (1990).

[45] J.G. Cordey et al., Nucl. Fusion 35, 101 (1995).

[46] K. Itoh and S.-I. Itoh, Plasma Phys. Control. Fusion 38, 1 (1996).

[47] K. Itoh, S.-I. Itoh and A. Fukuyama, Transport and Structural Formation in Plasmas (IOP, England, 1999). 
[48] K. Hallatschek, Plasma Phys. Control. Fusion 49, B137 (2007).

[49] M.N. Rosenbluth and F.L. Hinton, Phys. Rev. Lett. 80, 724 (1998).

[50] A. Fujisawa et al., Plasma Phys. Control. Fusion 48, S205 (2006).

[51] G.R. Hill et al., IAEA Fusion Energy Conf. (San Diego, 2012) OV/1-1.

[52] S-I. Itoh and K. Itoh, Plasma Phys. Control. Fusion 53, 015008 (2011).

[53] P.K. Kaw et al., Plasma Phys. Control. Fusion 44, 51 (2002).

[54] A. Fujiswa, A. Shimizu, H. Nakano et al., J. Phys. Soc. Jpn 76, 033501 (2007).

[55] S.-I. Itoh and K. Itoh, Plasma Phys. Control. Fusion 43, 1055 (2001).

[56] S. Inagaki et al., Phys. Rev. Lett. 107, 115001 (2011).

[57] S. Inagaki et al., Plasma Fusion Res. 6, 1402017 (2011).

[58] S. Inagaki et al., Nucl. Fusion 52, 023022 (2012).

[59] T.S. Hahm et al., Plasma Phys. Control. Fusion 46, A323 (2004).

[60] O.D. Gurcan, P.H. Diamond, T.S. Hahm and Z. Lin, Phys. Plasmas 12, 032303 (2005).

[61] X. Garbet et al., Phys. Plasmas 14, 122305 (2007).

[62] S. Sugita et al., Plasma Phys. Control. Fusion 54, 125001 (2012).

[63] U. Stroth, IAEA Fusion Energy Conf. (San Diego, 2012) OV $2 / 2$.

[64] K.C. Shaing et al., Phys. Rev. Lett. 63, 2369 (1989).

[65] S-I. Itoh and K. Itoh, Nucl. Fusion 29, 1031 (1989).

[66] S-I. Itoh, K. Itoh and S. Toda, Phys. Rev. Lett. 89, 215001 (2002).

[67] K. Kamiya et al., Phys. Rev. Lett. 105, 045004 (2010).

[68] K. Miki et al., Phys. Rev. Lett. 110, 195002 (2013).

[69] S.-I. Itoh and K. Itoh, 'Physics of L-H Transition and the Threshold Condition', presented at 14th International Workshop on H-mode Physics and Transport Barriers (Kasuga, Japan, 2013) (P02-18).

[70] M. Yagi, K. Shimizu, T. Takizuka, M. Honda, N. Hayashi, K. Hoshino and A. Fukuyama, Contrib. Plasma Phys. 52, 372 (2012).

[71] M. Honda, S. Ide, T. Takizuka, N. Hayashi, M. Yoshida, M.
Yagi and T. Fujita, Nucl. Fusion 53, 073050 (2013).

[72] M. Honda and A. Fukuyama, J. Comput. Phys. 227, 2808 (2008).

[73] A. Fukuyama, R. Kanamori and M. Honda, 'Transport modeling of rotation and barrier formation in multi-ionspecies plasmas', presented at 14th International Workshop on H-mode Physics and Transport Barriers (Kasuga, Japan, 2013) (P02-09).

[74] L. Chôné, P. Beyer, Y. Sarazin, G. Fuhr, C. Bourdelle and S. Benkadda, 'Spontaneous barrier formation in fluid turbulence simulations with neoclassical force balance', presented at 14th International Workshop on H-mode Physics and Transport Barriers (Kasuga, Japan, 2013) (P02-01).

[75] G.Y. Park, S.S. Kim, T. Rhee, H.G. Jhang, P.H. Diamond and X.Q. Xu, 'BOUT++ flux-driven simulation of edge transport barrier formation with sheared equilibrium flows', presented at 14th International Workshop on H-mode Physics and Transport Barriers (Kasuga, Japan, 2013) (P02-12).

[76] A. Strugarek et al., Phys. Rev. Lett. 111, 145001 (2013).

[77] K.C. Shaing, R.D. Hazeltine and H. Sanuki, Phys. Fluids B 4, 404 (1992).

[78] N. Kasuya and K. Itoh, Phys. Rev. Lett. 94, 195002 (2005).

[79] N. Kasuya and K. Itoh, Plasma Phys. Control. Fusion 48, A319 (2006).

[80] M. Honda, A. Fukuyama and N. Nakajima, J. Phys. Soc. Jpn. 80, 114502 (2011).

[81] R.J. Taylor, M.L. Brown, B.D. Fried et al., Phys. Rev. Lett. 63, 2365 (1989).

[82] R.R. Weynants, G. Van Oost, G. Bertschinger et al., Nucl. Fusion 32, 837 (1992).

[83] T.E. Stringer, Nucl. Fusion 33, 1249 (1993).

[84] K. Itoh, S.-I. Itoh, M. Yagi and A. Fukuyama, Phys. Plasmas 5, 4121 (1998).

[85] N. Kasuya, K. Itoh and Y. Takase, Plasma Phys. Control. Fusion 46, A235 (2004).

[86] T. Tokuzawa et al., 'Characteristics of between radial electric field and density fluctuation structures in transition control experiments in LHD', presented at 14th International Workshop on H-mode Physics and Transport Barriers (Kasuga, Japan, 2013) (P02-14).

[87] Y. Nagashima et al., Phys. Plasmas 16, 020706 (2009). 